\title{
The Joint WHO/FAO Expert Consultation on diet, nutrition and the prevention of chronic diseases: process, product and policy implications
}

\author{
Chizuru Nishida ${ }^{1, *}$, Ricardo Uauy ${ }^{2,3}$, Shiriki Kumanyika ${ }^{4}$ and Prakash Shetty ${ }^{5}$ \\ ${ }^{1}$ Department of Nutrition for Health and Development, WHO, Geneva, Switzerland: ${ }^{2}$ Institute of Nutrition and Food \\ Technology, University of Chile, Santiago, Chile: ${ }^{3}$ Department of Epidemiology and Population Health, London \\ School of Hygiene and Tropical Medicine, London, UK: ${ }^{4}$ Department of Biostatistics and Epidemiology, School of \\ Medicine, University of Pennsylvania, Philadelphia, PA, USA: ${ }^{5}$ Nutrition Planning, Assessment and Evaluation \\ Service, Food and Nutrition Division, FAO, Rome, Italy
}

The Joint WHO/FAO Expert Consultation on diet, nutrition and the prevention of chronic diseases took place in Geneva from 28 January to 1 February 2002. The overall objective of the Consultation was to review and update current international recommendations on diet, nutrition and the prevention of chronic diseases by evaluating the latest scientific evidence and lessons learned from implementing national intervention strategies to reduce the burden of these diseases. Specific objectives included:

1. Reviewing the changes in the dietary and health patterns worldwide and examining their relationship to emergence of chronic diseases, particularly obesity, type 2 diabetes, hypertension and cardiovascular diseases, cancer, dental disease, and osteoporosis.

2. Reviewing the latest scientific evidence on the relationship between diet, nutrition and chronic diseases.

3. Examining diet, nutrition and health issues from a life course perspective.

4. Considering gene/nutrient interactions and their implications.

5. Formulating recommendations concerning diet and nutrition for the prevention of chronic diseases to assist countries in developing and implementing effective evidence-based multisectoral policies and strategies.

6. Identifying further research needs.

The selection of the expert participants followed standard criteria, including geographic and sex balance, in consultation with regional offices. Thirty experts were identified, half from developing countries and countries in economic transition where diet-related chronic diseases are an increasing public health problem. Before being officially invited to participate, all were requested to declare possible conflicts of interest to ensure the quality and neutrality of each expert's contributions.

Four working groups composed of world-renowned experts prepared background papers on major diet-related chronic diseases, such as obesity, type-2 diabetes, cardiovascular diseases and cancer, that included current trends for each disease, a review of the strength and weakness of the scientific evidence linking diet and disease, and its policy implications. Other experts were responsible for papers on dental diseases and osteoporosis while WHO staff prepared a paper on the life course approach and FAO staff prepared a review of the global and regional food consumption patterns and trends. Each background paper was peer-reviewed; papers were submitted, together with comments by peer-reviewers (some 20 in all), to the Expert Consultation as a general framework for discussion.

\section{Report preparation}

Following round-table consultations with nongovernmental organizations and industry associations in April 2002 to obtain feedback on the Expert Consultation's initial draft report, the draft report was made available on WHO's website for review and comment by all interested parties until June 2002.

In August 2002, the Consultation's Chairperson, ViceChairperson and rapporteurs met in Geneva with the WHO/FAO secretariat members as well as with several experts on physical activity to review more than 120 sets of comments from governments of FAO/WHO Member States, representatives of the scientific community, nongovernmental organizations, commercial enterprises and interested individuals. These comments were likewise posted in their entirety on WHO's website for public scrutiny. In October 2002, several members of the Expert Consultation met with the Chairperson and the WHO/FAO Secretariat to finalize several pending issues. In December 2002 , the final draft report was sent to the members of the Expert Consultation for review and final approval. The report was then finalized and published in the WHO Technical Report Series (TRS 916) ${ }^{1}$ and formally launched by the Directors-General of FAO and WHO in Rome on 23 April 2003. 


\section{What is new about the report of the Joint WHO/FAO Expert Consultation}

The report of the Joint WHO/FAO Expert Consultation ${ }^{1}$ updates the report of the 1989 WHO Study Group ${ }^{2}$. The main differences in the content and approach taken in each report may be described as follows.

\section{Collaboration between health and agriculture sectors}

As the objectives of the Expert Consultation stated, its work built on that of the 1989 WHO Study Group on diet, nutrition and prevention of noncommunicable diseases ${ }^{2}$ that had been convened to review the scientific evidence on the relationship between diet, nutrition and chronic disease and to make recommendations on the prevention of chronic diseases, in particular, from the perspective of diet and nutrition. Although the 1989 Study Group recognized the need for an intersectoral approach to address the issue of food supply, the 2002 Expert Consultation was the first time, since the 1992 International Conference on Nutrition, that WHO and FAO jointly addressed diet, nutrition and the prevention of chronic diseases. As a consequence, the report of the Expert Consultation includes a chapter that examines global and regional food consumption patterns and trends. It also addresses in a preliminary manner the likely implications of nutrient recommendations and dietary guidelines for food supply and production and the need for developing integrated action strategies.

\section{Physical activity}

The primary purpose of the Expert Consultation was to examine and develop recommendations for diet and nutrition in the prevention of chronic diseases. However, the Consultation explicitly recognized the need for adequate physical activity in the context of diet, nutrition and health, and that 'in order to achieve the best results in preventing chronic diseases, the strategies and policies ... must fully recognize the essential role of diet, nutrition and physical activity' ${ }^{1}$. The recommended goal of $60 \mathrm{~min}$ a day of moderate-intensity activity, such as walking, needed to maintain healthy body weight, particularly for people with sedentary occupations is different from the widely accepted public health recommendation of $30 \mathrm{~min}$ of moderate-intensity physical activity. The difference between these two recommendations is their focus. There is evidence that $30 \mathrm{~min}$ of moderate activity is sufficient for cardiovascular and metabolic health, but not for achieving all health benefits. Considering that obesity prevention is a crucial public health goal, the Expert Consultation recommended $60 \mathrm{~min}$ a day of moderateintensity activity as an appropriate population goal. However, it was further noted that this recommendation applied to sedentary people and that extra activity was not relevant for those already engaged in physically demanding occupational activities and domestic tasks requiring high energy expenditure.

Strength of evidence on dietary and lifestyle factors The report of the Expert Consultation includes the criteria used to describe strength of evidence (Table 1). These were based on the criteria used in the World Cancer Research Fund's report on food, nutrition and the prevention of cancer $^{3}$ modified to include results of controlled trials where relevant and available. Furthermore, the Consultation recognized the complex interaction between environmental factors that affect excess weight gain as an important contributing risk factor for many chronic diseases. In categorizing risks, the Consultation took into consideration consistent evidence on community and environmental factors which lead to behavioural changes and thereby modify risk.

Table 1 Criteria used to describe the strength of evidence ${ }^{1}$

\begin{tabular}{|c|c|}
\hline Convincing evidence & $\begin{array}{l}\text { Evidence is based on epidemiological } \\
\text { studies showing consistent associations } \\
\text { between exposure and disease, with little } \\
\text { or no evidence to the contrary. The avail- } \\
\text { able evidence is based on a substantial } \\
\text { number of studies including prospective } \\
\text { observational studies and where rel- } \\
\text { evant, randomized controlled trials of suf- } \\
\text { ficient size, duration and quality showing } \\
\text { consistent effects. The association } \\
\text { should be biologically plausible }\end{array}$ \\
\hline
\end{tabular}

Probable evidence Evidence is based on epidemiological studies showing fairly consistent associations between exposure and disease, but where there are perceived shortcomings in the available evidence or some evidence to the contrary, precluding a more definite judgment. Shortcomings in the evidence may be any of the following: insufficient duration of trials (or studies); insufficient trials (or studies) available; inadequate sample sizes; and incomplete follow-up. Laboratory evidence is usually supportive. Again, the association should be biologically plausible

Possible evidence Evidence is based mainly on the findings from case-control and cross-sectional studies. Insufficient randomized controlled trials, observational studies or nonrandomized controlled trials are available. Evidence based on nonepidemiological studies, such as clinical and laboratory investigations, is supportive. More trials are required to support the tentative associations, which should also be biologically plausible

Insufficient evidence Evidence is based on the findings of a few studies which are suggestive, but are insufficient to establish an association between exposure and disease. Limited or no evidence is available from randomized controlled trials. More welldesigned research is required to support the tentative associations 


\section{Life course approach}

The Expert Consultation's application of a life course approach is in recognition of the fact that both undernutrition and overnutrition play a role in the development of chronic diseases. There is increasing evidence that the influences contributing to chronic disease development are part of a continuum, i.e. risks begin in fetal life and continue into old age. Chronic disease in adulthood thus reflects cumulative differential life time exposures to various damaging environments, whether physical, social or environmental. The Expert Consultation adopted a life course approach that captures both the cumulative risk and the many opportunities for interventions. For convenience 'life course' was divided into fetal development and the maternal environment, infancy, childhood and adolescence, adulthood, and ageing and old age.

\section{Changes in the values of several dietary components}

Table 2 lists the recommended goals of the 1989 WHO Study Group and those of the Joint WHO/FAO Expert Consultation together with the rationale for the changes, i.e. the list of diseases that contributed to those changes. The following are some of the highlights.
1. Detailed breakdown of the goals for fat. Providing greater detail on recommendations for fatty acids results from the Expert Consultation's recognition of the growing evidence that the qualitative composition of fats in the diet has a significant role to play in modifying the risk of cardiovascular diseases. To promote cardiovascular health, diets should provide very low intake (i.e. $<1 \%$ ) of trans fatty acids (hydrogenated fats). This recommendation is extremely important in many developing countries where low-cost hydrogenated fat is frequently consumed. Diet should also provide adequate intake of polyunsaturated fatty acids (PUFAs) (i.e. 6-10\% of daily energy intake). Furthermore, there should be an optimal balance between intake of $n$-6 PUFAs (5-8\% of daily energy intake) and $n$-3 PUFAs (1-2\% of daily energy intake).

2. Lowering of the goal for sodium chloride. Lowering the recommended value for sodium chloride was also related to the prevention of cardiovascular diseases. High blood pressure is a major risk factor for coronary heart disease and both forms of stroke, i.e. ischemic and hemorrhagic, and dietary intake of sodium influences blood pressure levels in populations. Current evidence suggests that an intake of no more

Table 2 Ranges of population nutrient intake goals (\% of total energy, unless otherwise stated)

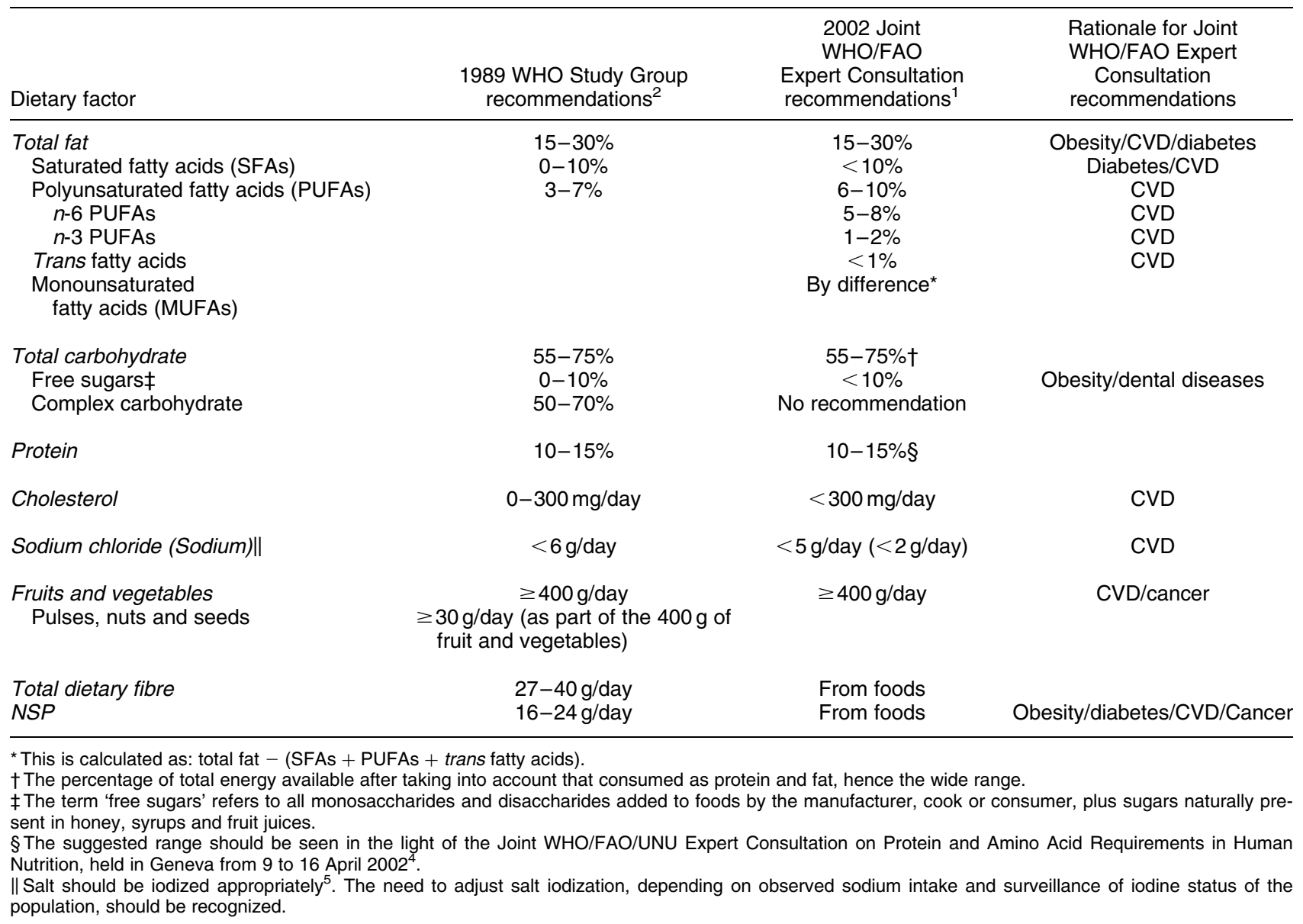


than $70 \mathrm{mmol}$ or $1.7 \mathrm{~g}$ of sodium per day is beneficial in reducing blood pressure. The recommended daily salt (sodium chloride) intake is thus less than $5 \mathrm{~g}$. However, given the level of iodine deficiency disorders in some countries, the Expert Consultation emphasized the need to adjust salt iodization ${ }^{5}$ depending on observed sodium intake and surveillance of iodine status of the concerned populations.

3. Dietary fiber/NSP from foods. Although a definition for dietary fiber has not yet been established, the Expert Consultation report expresses dietary fiber as nonstarch polysaccharides (NSP). Current evidence indicates that adequate NSP intakes through regular consumption of whole-grain cereals, legumes, fruits and vegetables have potential health benefits, in particular for preventing obesity, diabetes, cardiovascular diseases and various cancers. The Expert Consultation, therefore, agreed that the recommended intake of fruits and vegetables, i.e. $\geq 400 \mathrm{~g} / \mathrm{d}$, and consumption of whole-grain foods is likely to provide $>20 \mathrm{~g} / \mathrm{d}$ of NSP, i.e. $>25 \mathrm{~g} / \mathrm{d}$ of total dietary fiber.

4. Free sugars. The recommendation for free sugars, i.e. $<10 \%$ of energy, is slightly different from the recommendation made by the 1989 WHO Study Group $^{2}$ in that it provides no lower limit. The Joint WHO/FAO Expert Consultation was aware of the controversy surrounding a population goal for free sugars of $<10 \%$ of total energy, but it also considered that studies showing no effect of free sugars on excess weight have significant limitations.

At any given level of energy intake, higher intakes of free sugars threaten the nutrient quality of diets by providing significant amounts of energy without supplying specific nutrients ${ }^{6-9}$. The Expert Consultation agreed that restriction of free sugars was also likely to contribute to reducing the risk of unhealthy weight gain in the light of the following considerations:

- Free sugars contribute to the overall energy density of diets.

- Free sugars promote a positive energy balance. Research in human volunteers demonstrates increased total energy intake in short-term studies when dietary energy density is increased, whether by free sugars or fat. Diets that are limited in free sugars have been shown to reduce total energy intake and induce weight loss ${ }^{10-13}$.

- Drinks that are rich in free sugars increase overall energy intake by reducing appetite control. There is thus less of a compensatory reduction of food intake after the consumption of high-sugar drinks than when additional foods of equivalent energy content are provided. A recent randomized trial showed that when free sugar-rich soft drinks are consumed energy intake is higher and there is a progressive increase in body weight compared with energy-free drinks that are artificially sweetened ${ }^{14}$. Children who consume large quantities of soft drinks rich in free sugars are more likely to be overweight and to gain excess weight ${ }^{15}$.

The US Institute of Medicine (IOM) of the National Academies also made similar analyses in their report ${ }^{16}$. However, the $25 \%$ figure cited in the IOM report has been misinterpreted and misused by some to criticize the Expert Consultation's $<10 \%$ recommendation despite the fact that IOM's recommendation of $25 \%$ does not represent a desirable or even acceptable standard sugar intake. It is rather a suggested maximum intake based on the decreased intake of some micronutrients of American sub-populations, and is not related to weight gain or obesity.

Contribution to national strategy and policy development The report of the 1989 WHO Study Group (TRS 797) ${ }^{2}$ served as a reference for developing the strategy for promoting healthy diets and lifestyles in the World Declaration and Plan of Action for Nutrition ${ }^{17}$ adopted at the International Conference on Nutrition in $1992^{18,19}$ and endorsed by the World Health Assembly in $1993^{20}$. Thanks to the efforts of national governments and the international community to implement the Declaration and Plan of Action, by November 2003, 146 out of WHO's 192 Member States had developed and strengthened their national food and nutrition plans and policies ${ }^{21}$. At least 100 of these countries (and 5 territories) had incorporated strategies for promoting healthy diets and lifestyles in their national food and nutrition plans and policies ${ }^{21}$. Today, the updated goals recommended by the Joint WHO/FAO Expert Consultation provide an important scientific basis for developing and implementing global, regional and national strategies for improving health and nutritional well-being of the world population.

\section{Population nutrient intake goals}

The Expert Consultation's nutrient recommendations are population intake goals, not individual dietary guidelines. The concept of population nutrient intake goals is based on the assumption that the first priority is to ensure national food security and equity of distribution of available food in accordance with individual needs.

Most nutritional guidelines address the estimated needs of individuals and identify the minimum intake to meet the nutritional needs of individuals. However, in recognition of the detrimental effects that excessively high intakes of essential nutrients may have, the concept of a safe range of intakes has evolved. Population nutrient intake goals follow this concept and focus on the maintenance of low population risk rather than low individual risk. Population nutrient intake goals are thus defined as the population average intake that is judged to be consistent with maintenance of health in a population. Health, in this context, is marked by a low prevalence of diet-related 
diseases in the population. ${ }^{, 12}$. In other words, it is the entire distribution of intakes, characterized by the average per capita intake, that is of interest and not the intakes of individuals.

The Joint WHO/FAO Expert Consultation stressed that because population nutrient intake goals refer to population averages, the aggregate change would be substantially greater than intended if they were to be applied to the diets of individuals. This point is illustrated in Fig. 1 using the Consultation's intake recommendations for free sugars and saturated fatty acids (SFAs). Moreover, the intention is for population nutrient intake goals to be adapted to diets and populations by taking into account local circumstances. These goals are expressed in numerical terms rather than as increases or decreases in intakes of specific nutrients since the desirable up or down change depends on existing intakes in a given population.

\section{Implementation process}

According to the WHO Global Database on National Nutrition Policies and Programs ${ }^{21}, 94$ countries and 6 territories have developed or revised national dietary guidelines, often in direct response to the World Declaration and Plan of Action for Nutrition ${ }^{17}$. The Plan of Action also called for dissemination of nutrition information through 'sustainable food-based approaches that encourage dietary diversification through the production and consumption of micronutrient-rich food, including appropriate traditional foods ${ }^{17}$.

In 1995, FAO and WHO jointly organized an Expert Consultation on development and use of food-based dietary guidelines (FBDGs ${ }^{22}$. Using this global framework, some WHO regional offices then prepared regional

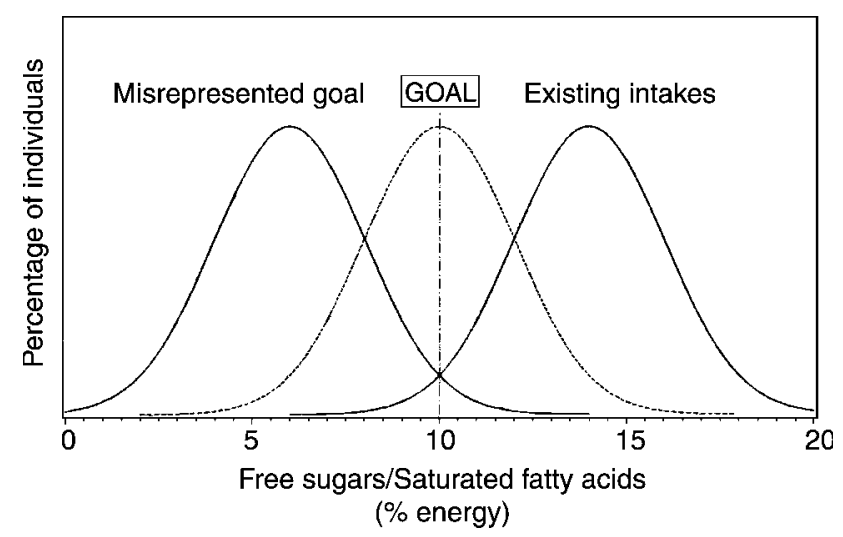

Fig. 1 Distinction between a population goal and an individual goal. This figure shows the distribution of free sugars and SFAs for three hypothetical populations, corresponding to: (1) an existing population whose intake of free sugars and SFAs is higher than recommended by the joint WHO/FAO Expert Consultation (right); (2) a population that has achieved the population goal of the maximum intake of $10 \%$ of energy as free sugars and SFAs (centre); and (3) a population in which nearly all individuals have an intake less than $10 \%$, a situation that describes the result of misrepresentation of the population goal as an individual goal (left) (adapted from the report of the WHO Study Group ${ }^{2}$ ).
FBDGs, which take into consideration public health concerns, food availability, tradition and culture and the environment $^{23-26}$. Based on these global and regional guidelines, to date 81 out of the above-mentioned 94 countries and 6 territories have developed national FBDGs $^{21}$. In the light of the updated population nutrient intake goals recommended by the Joint WHO/FAO Expert Consultation, national FBDGs should be reviewed or, as necessary, formulated by adapting recommended population nutrient intake goals to local situations.

Ideally, population nutrient intake goals and energy and nutrient requirements (including for micronutrients) will be taken into account when formulating national food and nutrition policies. Currently, a number of initiatives are being implemented that seek to place diet and nutrition high on the political agenda. These include development of global and regional strategies, such as for infant and young child feeding; child and adolescent health and development; diet, physical activity and health; and food safety, a call-to-action for an integrated multisectoral approach to preventing and controlling obesity in the Pacific, Tonga commitment to promote healthy lifestyles and supportive environment by the health ministers and the Regional Food and Nutrition Action Plan for Europe, to mention just a few.

The challenges are many in the continuing struggle to eliminate hunger and malnutrition while responding to the emerging public health challenge of diet-related chronic diseases. Accelerating implementation of effective food and nutrition policies and strategies requires firm political commitment and ground-breaking interaction among all concerned stakeholders.

\section{Acknowledgements}

Summaries of the information contained in this paper were presented by the authors at the Working Group on Nutrition Throughout the Life Cycle held during the 30th Session of the UN System Standing Committee on Nutrition (SCN) in Chennai, India, in March 2003 as well as at the official launching of the report of the Joint WHO/FAO Expert Consultation held in Rome in April 2003. The authors wish to thank Professor Jim Mann, Department of Human Nutrition, University of Otago, Dunedin, New Zealand, and colleagues from the Department of Nutrition for Health and Development (NHD), WHO, Geneva, Switzerland, including Dr Bruno de Benoist, Director, a.i., Dr Graeme Clugston, former Director, and Mr James Akré, for their valuable comments and advice on the earlier manuscript.

\section{References}

1 Diet, Nutrition and the Prevention of Chronic Diseases. Report of a Joint WHO/FAO Expert Consultation. WHO Technical Report Series No. 916. Geneva: World Health Organization, 2003. 
2 Diet, Nutrition, and the Prevention of Chronic Diseases. Report of a WHO Study Group. WHO Technical Report Series No. 797. Geneva: World Health Organization, 1990.

3 World Cancer Research Fund. Food, Nutrition and the Prevention of Cancer: A Global Perspective. Washington, DC: American Institute for Cancer Research, 1997.

4 Protein and Amino acid Requirements in Human Nutrition. Report of a Joint WHO/FAO/UNU Expert Consultation. Geneva: World Health Organization, 2004 (In press).

5 WHO/UNICEF/ICCIDD. Recommended Iodine Levels in Salt and Guidelines for Monitoring Their Adequacy and Effectiveness. Geneva: World Health Organization, 1996 (document WHO/NUT/96.13).

6 Steyn NP, Myburgh NG, Nel JH. Evidence to support a foodbased dietary guideline on sugar consumption in South Africa. Bulletin of the World Health Organization 2003; 81: 599-608.

7 Mann J. Sugar revisited-again. Bulletin of the World Health Organization 2003; 81: 552-3.

8 Gay J, et al. Dietary factors in epidemic neuropathy on the Isle of Youth, Cuba. Bulletin of the Pan American Health Organization 1995; 29: 25-36.

9 Roman GC. An epidemic in Cuba of optic neuropathy, sensorineural deafness, peripheral sensory neuropathy and dorsolateral myeloneuropathy. Journal of the Neurological Sciences 1994; 127: 11-28.

10 Mann JI, et al. Effects on serum-lipids in normal men of reducing dietary sucrose or starch for five months. Lancet 1970; 1: 870-2.

11 Smith JB, Niven BE, Mann JI. The effect of reduced extrinsic sucrose intake on plasma triglyceride levels. European Journal of Clinical Nutrition 1996; 50: 498-504.

12 Saris WH, et al. Randomized controlled trial of changes in dietary carbohydrate/fat ratio and simple vs complex carbohydrates on body weight and blood lipids: the CARMEN study. The Carbohydrate Ratio Management in European National diets. International Journal of Obesity and Related Metabolic Disorders 2000; 24: 1310-8.

13 Poppitt SD, et al. Long-term effects of ad libitum low-fat, high-carbohydrate diets on body weight and serum lipids in overweight subjects with metabolic syndrome. American Journal of Clinical Nutrition 2002; 75: 11-20.

14 Raben A, et al. Sucrose compared with artificial sweeteners different effects on ad libitum food intake and body weight after 10 wk of supplementation in overweight subjects. American Journal of Clinical Nutrition 2002; 76: 721-9.

15 Ludwig DS, Peterson KE, Gormakaer SL. Relation between consumption of sugar-sweetened drinks and childhood obesity: a prospective, observational analysis. Lancet 2001; 357: $505-8$

16 Institute of Medicine of the National Academies. Dietary Reference Intakes: Energy, Carbohydrate, Fiber, Fatty acids, Cholesterol, Protein, and Amino acids. Washington, DC: The National Academies Press, 2002 (Prepublication copy).

17 World Declaration and Plan of Action for Nutrition. Rome, Food and Agriculture Organization of the United Nations and Geneva, World Health Organization, 1992.

18 Nutrition and Development: A Global Assessment. Rome, Food and Agriculture Organization of the United Nations and Geneva, World Health Organization, 1992.

19 Promoting appropriate diets and healthy lifestyles. In: Major Issues for Nutrition Strategies. Rome, Food and Agriculture Organization of the United Nations and Geneva, World Health Organization, 1992.

20 Resolution WHA 46.7. International Conference on Nutrition: follow-up action. Forty-sixth World Health Assembly, Geneva, 3-14 May 1993. Volume 1. Resolutions and Decisions, Annexes. Geneva: World Health Organization, 1993 (document WHA46/1993/REC/1).

21 WHO Global Database on National Nutrition Policies and Programmes. Geneva: Department of Nutrition, World Health Organization, 2003.

22 Preparation and Use of Food-based Dietary Guidelines. Report of a Joint FAO/WHO Consultation. WHO Technical Report Series, No. 880. Geneva: World Health Organization, 1998.

23 Peña M, Molina V. Food Based Dietary Guidelines and Health Promotion in Latin America. Washington, DC: Pan American Health Organization and Institute of Nutrition of Central America and Panama, 1999.

24 CINDI Dietary Guidelines. Copenhagen: World Health Organization, 2000.

25 Development of Food Based Dietary Guidelines for the Asian region. Final report of the recommendations of the Asian Nutrition Forum/WHO Symposium on Diet Related Chronic Diseases in Asia, New Delhi, India International Centre, 10-14 February 1997.

26 Development of Food-based Dietary Guidelines for the Western Pacific Region: The Shift from Nutrients and Food Groups to Food Availability, Traditional Cuisine and Modern Foods in Relation to Emerging Chronic Noncommunicable Diseases. Manila: WHO Regional Office for the Western Pacific, 1999. 